\title{
Infections in Cancer Patients
}

\author{
Deepjot Singh and Robert A. Bonomo
}

Additional information is available at the end of the chapter

http://dx.doi.org/10.5772/64372

\begin{abstract}
Cancer therapy is a dynamically evolving field. Chemotherapy and biologic agents impact the magnitude and duration of immunosuppression in the already-immunocompromised cancer hosts who are then susceptible to a broad spectrum of infectious complications ranging from mild opportunistic infections to severe, fatal neutropenic sepsis. Numerous bacterial, fungal, and viral organisms have been implicated dictating varied preventative approaches. Rapid assessment and risk stratification of febrile patients identify individuals requiring hospital admission. Timely delivery of antimicrobials reduces the risk of complications and death. Herein, we summarize the current "state of art" in the management of infection in the cancer patient. We detail the advances in antibacterial and antifungal therapy.
\end{abstract}

Keywords: Cancer, Fever, Infection, Neutropenia, Chemotherapy

\section{Introduction}

Cancer patients are at a risk for development of severe infections. Predisposing factors include severe neutropenia, impaired neutrophil function, and B-cell, T-cell, or NK-cell defects. Patients with chronic lymphocytic leukemia often have hypogammaglobulinemia which increases susceptibility to encapsulated bacteria. Patients with advanced solid tumors including head and neck cancer, lung cancer, gastrointestinal malignancy, and pancreatic cancer are commonly malnourished. Malnutrition impairs immune function and increases the susceptibility to infection. Chemotherapy, biological agents, and high-dose steroids may also cause significant immunosuppression, thereby increasing the risk for infection. In the United States, approximately 60,000 patients are admitted due to neutropenia, annually. One in 
fourteen of these patients dies secondary to sepsis-related complications [1]. The estimated daily cost of hospitalization is $\$ 2,000-\$ 3,000$ [2]. Advances in infection control and antimicrobial stewardship are therefore important to diminish the impact of infections in these immunocompromised hosts.

\section{Cancer therapy-related infections}

Most single chemotherapeutic agents used for the treatment of solid tumors do not cause prolonged neutropenia and are therefore associated with a low risk for bacterial infections. Additionally, they do not cause significant suppression of T-cell function leading to clinically relevant viral reactivation. On the other hand, induction and consolidation chemotherapy for acute leukemia may result in severe, prolonged neutropenia, thereby not only increasing the risk of bacterial and fungal infections but also predisposing to herpes simplex virus (HSV) reactivation [3].

Epidermal growth factor receptor (EGFR) inhibitors are commonly used in the treatment of solid tumors. Cetuximab is a chimeric murine-human IgG1 monoclonal antibody that is used in the treatment of head and neck cancer and advanced colorectal cancer. Panitumumab is a fully human IgG2 monoclonal antibody used in the treatment of advanced colorectal cancer. Erlotinib and gefitinib are used in the treatment of lung adenocarcinoma. These EGFR inhibitors may be associated with acneiform eruptions and paronychia. Severe skin toxicities complicated by infection, abscess, and sepsis have also been reported. Trastuzumab and pertuzumab are anti-HER2 monoclonal antibodies used in the treatment of breast cancer. In a meta-analysis of 13 randomized studies of breast cancer patients $(N=10,094)$, treatment with trastuzumab was associated with $8.5 \%$ (95\% CI 4.5-15.4\%) incidence of high-grade infection and $12.0 \%$ (95\% CI 8.1-17.4\%) incidence of febrile neutropenia in the absence of high-grade neutropenia or leukopenia [4].

Mammalian target of rapamycin (mTOR) inhibitors (temsirolimus and everolimus) is used in the treatment of advanced renal cell carcinoma. Severe lymphopenia, neutropenia, and sepsis have been reported. Immunosuppression with these agents may increase the risk of opportunistic infections including Pneumocystis jiroveci pneumonia (PJP). In a meta-analysis of ten randomized trials of cancer patients $(N=3,535)$, everolimus and temsirolimus were associated with a $21 \%(95 \%$ CI 15.0-28.9\%) risk of high-grade infections [5].

Vascular endothelial growth factor receptor/tyrosine kinase inhibitors (VEGFR-TKIs) block angiogenesis by inhibiting VEGF and other growth factors. They are used in the treatment of a variety of tumors including chronic myelogenous leukemia (imatinib, dasatinib, nilotinib), renal cancer (sunitinib, sorafenib, pazopanib, and axitinib), hepatocellular carcinoma (sorafenib), colorectal cancer (regorafenib), thyroid cancer (sorafenib, vandetanib), and sarcoma (cediranib). In a meta-analysis of 27 randomized trials $(N=16,488)$, VEGFR-TKIs significantly increased the risk of developing severe (1.69-fold) and fatal infectious events (1.78-fold) in cancer patients [6]. 
Monoclonal antibodies that target B lymphocytes cause significant cellular immunosuppression predisposing to bacterial, fungal, and viral infections. Reactivation of hepatitis B virus (HBV) is more common compared to other viruses such as herpes simplex virus (HSV), varicella zoster virus (VZV), cytomegalovirus (CMV), and Epstein-Barr virus (EBV) [7]. These agents should not be administered to patients with active infection. Anti-CD20directed monoclonal antibodies (rituximab, ofatumumab, and obinutuzumab) are used in the treatment of lymphoproliferative disorders. Their use is associated with HBV reactivation resulting in fulminant hepatitis, hepatic failure, and death [8,9]. Anti-CD30-directed monoclonal antibody (brentuximab vedotin) is used in the management of relapsed Hodgkin's lymphoma and anaplastic large-cell lymphoma. Its use is associated with prolonged severe neutropenia and neutropenic fever. Cases of progressive multifocal leukoencephalopathy (PML) and death due to JC virus infection have also been reported [10]. Anti-CD38-directed monoclonal antibody (daratumumab) is used in the management of refractory multiple myeloma. Its use is associated with myelosuppression. Herpes zoster occurs in $3 \%$ of patients. Antiviral prophylaxis to prevent herpes zoster reactivation should be initiated within one week of starting daratumumab and continued for three months following the last dose [11]. Anti-CD52-directed monoclonal antibody (alemtuzu$\mathrm{mab}$ ) is used for treatment of chronic lymphocytic leukemia. It induces severe and prolonged lymphopenia and increases the risk of serious and potentially fatal bacterial, viral, fungal, and protozoan infections. Prophylactic medications against PJP and herpes virus infection during treatment and for at least 2 months following last dose or until CD4+ counts are $\geq 200$ cells $/ \mu \mathrm{L}$ are recommended. Close monitoring for CMV reactivation is also recommended [12].

Signaling lymphocyte activation molecule family 7 (SLAMF7) is present on myeloma cells and natural killer cells. Anti-SLAMF7-directed monoclonal antibody (elotuzumab) is administered in combination with lenalidomide and dexamethasone for refractory myeloma. Bone marrow suppression and increased risk of opportunistic, fungal, and herpes zoster infection have been reported [13].

Janus kinase (JAK) 1/2 inhibitor, ruxolitinib, is used for the treatment of polycythemia vera and myelofibrosis. Its use is associated with impairment of dendritic cell and T-cell function and reduction in cytokines resulting in the development of serious bacterial, fungal, and viral infections. Opportunistic infections reported include HBV reactivation, disseminated tuberculosis, Cryptococcus pneumonia, toxoplasmosis retinitis, and PML [14, 15].

Checkpoint inhibitors target cytotoxic T-lymphocyte-associated antigen 4 (CTLA-4) and programmed cell death-1 (PD-1) receptor. Anti-CTLA-4 antibody (ipilimumab) is used in the treatment of melanoma. Anti-PD-1 antibodies (nivolumab and pembrolizumab) are used in the management of melanoma and lung cancer. Checkpoint inhibition is associated with immune-mediated adverse events including pneumonitis, colitis, hepatitis, nephritis, dermatitis, and endocrinopathies. High-dose glucocorticoids are used in the management of grade 2 or greater immune-related toxicities, which increase the risk for development of opportunistic infections. 


\section{Role of antimicrobial prophylaxis in the prevention of cancer-related infections}

Infection risk in patients with cancer depends on several factors including age more than 65 years, tumor burden (size and number of lesions), regimen and intensity of cytotoxic chemotherapy, duration of neutropenia, degree of mucositis, and associated comorbidities. Intense cytotoxic chemotherapy causes prolonged neutropenia which impairs the inflammatory response and predisposes the individual to serious infection(Figure 1).

Low risk (Anticipated neutropenia $<7$ days)

- Chemotherapy regimens for most solid tumors

Intermediate risk (Anticipated neutropenia 7-10 days)

- Chemotherapy regimens for most hematologic malignancies

- Autologous hematopoietic stem cell transplant (HSCT)

High risk (Anticipated neutropenia $>10$ days)

- Chemotherapy for acute leukemia

- Allogeneic HSCT

- Alemtuzumab therapy

Figure 1. National Comprehensive Cancer Network (NCCN) criteria for risk of infection in patients undergoing chemotherapy [16].

Neutropenia is a major risk factor for the development of infections. Neutropenic fever is more common after chemotherapy for hematologic malignancies than solid tumors. Preventive measures against infections involve antimicrobial prophylaxis for patients receiving chemotherapy regimens associated with greater than or equal to twenty percent risk for fever and neutropenia.

\subsection{Antibacterial prophylaxis during neutropenia}

Patients receiving combination or dose-intensive chemotherapy are at increased risk for prolonged neutropenia [absolute neutrophil count (ANC) $<1,000 / \mu \mathrm{L}$ lasting more than 7 days] and bacterial infections. Gram-negative bacilli are associated with life-threatening infections. In a meta-analysis of neutropenic patients (18 trials, $N=1,408)$ with solid tumors and hematological malignancies, fluoroquinolone prophylaxis (ciprofloxacin, norfloxacin, enoxacin, and 
ofloxacin) significantly lowered the incidence of gram-negative infections by $80 \%$ (RR, 0.21 ; $95 \%$ CI 0.12-0.37) when compared with placebo and by $70 \%$ when compared with trimethoprim-sulfamethoxazole (TMP-SMX). Quinolone prophylaxis did not alter the incidence of gram-positive bacterial and fungal infections or infection-related deaths [17]. In another metaanalysis of afebrile neutropenic patients (109 trials, $N=13,579)$ with hematologic malignancies, antibiotic prophylaxis significantly decreased all-cause mortality (RR 0.66, $95 \%$ CI 0.55-0.79) and infection-related mortality (RR $0.61,95 \%$ CI $0.48-0.77$ ) compared to placebo or no intervention. Quinolone prophylaxis was associated with the most significant reduction in mortality [18]. Ciprofloxacin is more potent than levofloxacin against gram-negative bacteria. Nevertheless, levofloxacin with wider activity against gram-positive cocci may benefit as a prophylactic agent for mucositis-associated infections.

Levofloxacin prophylaxis in high-risk patients with anticipated prolonged neutropenia reduces clinically significant bacterial infections including gram-negative bacteremia [19]. Prophylaxis in low-risk patients with anticipated short-duration neutropenia decreases fever and hospitalization for febrile neutropenia, but not infection-related mortality [20]. Fluoroquinolone use may be associated with hypersensitivity reactions, prolonged QTc interval, tendon rupture, peripheral neuropathy, seizures, Clostridium difficile diarrhea, Streptococcus viridans bacteremia, and methicillin-resistant Staphylococcus aureus (MRSA) infection [21-23].

The National Comprehensive Cancer Network (NCCN) guidelines panel recommends the use of fluoroquinolone prophylaxis (levofloxacin) for patients with anticipated prolonged neutropenia. Antibacterial prophylaxis is not recommended for anticipated short-duration neutropenia due to the risk of emergence of quinolone-resistant bacteria [24].

\subsection{Antifungal prophylaxis}

Patients with acute myeloid leukemia (AML) and myelodysplastic syndrome (MDS) and allogeneic hematopoietic stem cell transplant (HSCT) recipients are at increased risk for lifethreatening infections with yeasts or molds. Candida and Aspergillus species are the most common pathogens. Amphotericin B (AMB) has activity against both Candida spp. and Aspergillus spp. but is too toxic for antifungal prophylaxis.

Studies supporting antifungal prophylaxis in patients undergoing induction chemotherapy for AML have shown that fluconazole is superior to placebo in preventing invasive candidiasis [25] and is as effective as amphotericin B [26]. However, fluconazole lacks activity against molds. Itraconazole has activity against both Candida and Aspergillus species. It significantly reduces invasive fungal disease (IFD) compared to fluconazole at the cost of greater toxicity [27-29]. Voriconazole is active against a wider range of fungi including Candida spp., Aspergillus spp., Scedosporium spp., and Fusarium spp. [30]. Nevertheless, data supporting voriconazole prophylaxis in the non-transplant AML population is limited. Posaconazole has broader antifungal coverage including Candida spp., Aspergillus spp., Scedosporium spp., Fusarium spp., and Mucorales. It is the only agent that has demonstrated survival benefit in prophylaxis against mycosis during AML induction therapy [31]. Azoles (itraconazole, voriconazole, and posaconazole) inhibit cytochrome P450 3A4 isoenzyme and may decrease the clearance of antineoplastic agent vincristine. Caspofungin is active against both Candida and Aspergillus spp. It has 
similar efficacy as itraconazole and is better tolerated [32]. It may therefore be a reasonable substitute for antifungal prophylaxis in patients who are unable to tolerate oral posaconazole.

Antifungal prophylaxis in patients undergoing HSCT is more complex due to concerns for poor oral absorption due to mucositis and drug interactions with antineoplastic and immunosuppressive medications. Fluconazole decreases invasive candidiasis and IFD-related mortality. Itraconazole has superior efficacy than fluconazole in prevention of IFD. Coadministration of itraconazole with antineoplastic conditioning regimens containing cyclophosphamide is associated with increased incidence of renal and hepatic toxicities. Voriconazole is as effective as itraconazole and is better tolerated [33]. Micafungin is as efficacious as itraconazole with less toxicity [34]. In patients with GVHD requiring immunosuppressive therapy, posaconazole has demonstrated improvement in IFD-related mortality ( $1 \%$ vs. $4 \%, p=.046)$ compared to fluconazole [35, 36] (Figure 2).

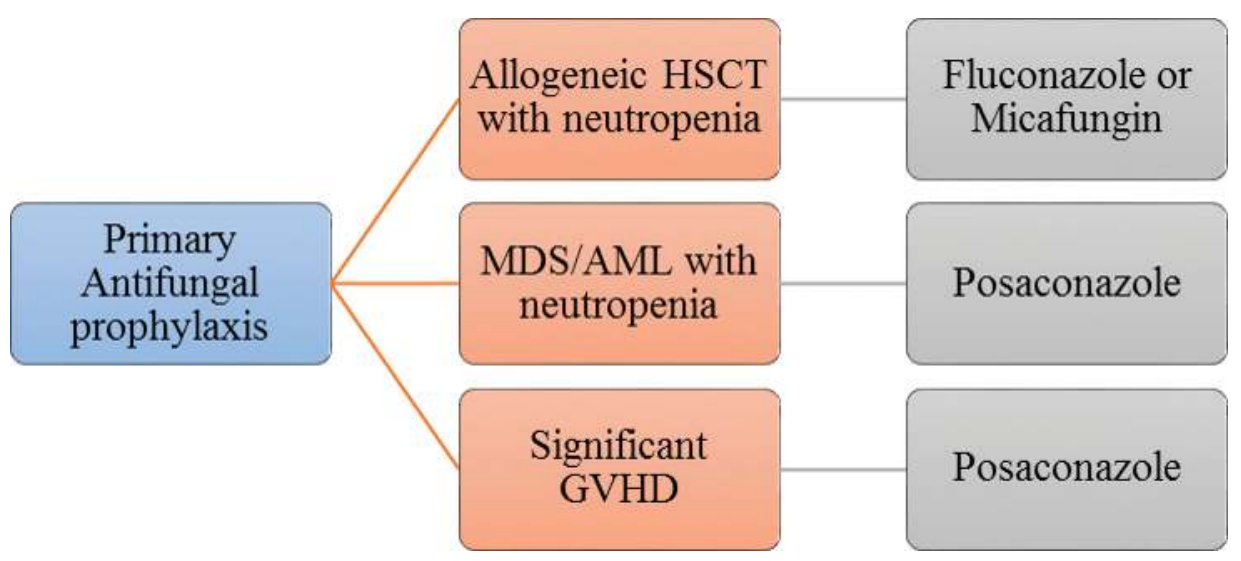

Figure 2. Primary antifungal prophylaxis in patients with hematologic disorders. HSCT, hematopoietic stem cell transplant; MDS, myelodysplastic syndrome; AML, acute myeloid leukemia; GVHD, graft-versus-host disease.

\subsection{Anti-pneumocystis prophylaxis}

P. jiroveci pneumonia (PJP) is a potentially life-threatening infection that may occur in immunocompromised individuals with acute lymphocytic leukemia (ALL) and recipients of allogeneic HSCT and alemtuzumab therapy. Patients receiving purine analog therapy, temozolomide in conjunction with radiation therapy, and high-dose glucocorticoids (equivalent to $\geq 20 \mathrm{mg}$ of prednisone daily for 4 or more weeks) may also be at risk.

In patients with AML or solid organ transplantation, prophylaxis with TMP/SMX compared to no treatment or treatment with fluoroquinolones reduced the incidence of PJP infections by $85 \%$ (RR 0.15, $95 \%$ CI 0.04-0.62; ten trials, 1,000 patients) and PJP-related mortality by $83 \%$ (RR 0.17, $95 \%$ CI 0.03-0.94; nine trials, 886 patients). Reduction in all-cause mortality was not observed. There was also no difference between once daily vs. thrice weekly TMP/SMX [37]. 
TMP/SMX is superior to dapsone and pentamidine in allogeneic HSCT recipients [38, 39]. PJP prophylaxis is administered for six months in allogeneic HSCT recipients and longer in patients with GVHD. ALL patients should receive prophylaxis till completion of immunosuppressive therapy. For those receiving alemtuzumab, anti-PJP prophylaxis is continued for a minimum of 2 months beyond alemtuzumab therapy or when the CD4+ cell count is above 200 cells/ $\mu \mathrm{L}$ [16].

\subsection{Antiviral prophylaxis}

Most cancer patients are at low risk of contracting viral infections. Immunosuppression may however predispose them to respiratory tract viral infections. Reactivation of HSV, VZV, and $\mathrm{HBV}$ is more likely to occur during intensive chemotherapy. EBV and CMV occur in the setting of allogeneic HSCT. The risk of viral infection increases with the intensity and duration of Tcell suppression. The extent of neutropenia is less important.

\subsubsection{Influenza virus}

Inactivated influenza vaccine is administered annually to patients undergoing chemotherapy [40]. Patients receiving induction or consolidation therapy for AML or those who have received anti-B-cell antibody within the last six months are excluded. Vaccination should be administered at least two weeks prior to receiving immunosuppressive therapy. Patients should be considered unprotected if they were vaccinated less than 2 weeks before start of immunosuppressive therapy. These patients should be revaccinated at least three months after the cytotoxic therapy is discontinued [41]. Acute leukemics should be vaccinated after completion of chemotherapy. Patients with ALL should receive the vaccine during the maintenance phase of their therapy [42].

\subsubsection{Hepatitis $B$ virus (HBV)}

Reactivation of latent HBV occurs in patients with leukemia (ALL, AML, and chronic lymphocytic leukemia (CLL)), lymphoma, myeloma, and breast cancer, transplant recipients, or patients receiving high-dose steroids, anti-CD 20 antibodies, alemtuzumab, or purine analogs. Screening tests include hepatitis B surface antigen (HBsAg), hepatitis B core antibody (anti-HBc), and HBV DNA. Patients who are HBsAg positive/anti-HBc positive or HBsAg negative/anti-HBc positive are at risk for reactivation. Antiviral therapy should be initiated in patients with HBsAg-positive/anti-HBc-positive serology either prior to or concurrent with cytotoxic therapy. HBsAg-negative/anti-HBc-positive patients may be monitored for reactivation with HBV DNA and Alanine Transferase (ALT) levels and antivirals initiated at reactivation. Evidence of reactivation includes change in the HBV DNA from undetectable to detectable, or $\geq 1 \log$ rise in HBV DNA level above baseline, or seroconversion from negative to positive HBsAg status. ALT should be monitored to assess hepatic function in the setting of HBV reactivation [43]. HBV DNA is monitored monthly during cytotoxic therapy and then every 3 months after completion of therapy. Antiviral therapy should be continued for 6 months after completion of cytotoxic therapy and for longer than 12 months in patients treated with anti-CD 20 monoclonal antibodies. HBV prophylaxis re- 
sults in $87 \%$ relative risk reduction of reactivation [43]. It also prevents fulminant hepatitis [44]. Entecavir is more effective than lamivudine and is associated with lower incidence of viral resistance and hepatitis [9]. Allogeneic HSCT candidates with evidence of active HBV infection should receive antiviral therapy for three to six months prior to initiation of conditioning [45].

\subsubsection{Herpes simplex virus (HSV)}

Reactivation and infection with HSV occur in patients undergoing induction therapy for acute leukemia and HSCT recipients. Prolonged neutropenia and mucositis are major predisposing factors. The risk of HSV reactivation is highest in the first 30 days following allogeneic transplant. Screening tests include HSV-1 and HSV-2 IgG antibodies. In a meta-analysis of nine randomized trials of HSCT recipients, acyclovir prophylaxis reduced HSV infection (RR 0.19, $95 \%$ CI 0.11-0.31) without impacting overall mortality [46]. Antiviral prophylaxis with acyclovir or valacyclovir is therefore recommended in HSV-seropositive patients. The antiviral agent is administered with the initiation of the conditioning regimen and is continued till either engraftment occurs or the mucositis has resolved. Patients with chronic lymphocytic leukemia (CLL) receiving alemtuzumab therapy are also at risk for HSV infection. Antiviral prophylaxis is recommended for 2 months beyond alemtuzumab therapy or when the CD4+ cell count is above 200 cells/ $\mu \mathrm{L}$ [47].

\subsubsection{Varicella zoster virus (VZV)}

Reactivation of VZV occurs in seropositive HSCT recipients. Screening test includes VZV IgG antibody. Antiviral prophylaxis with acyclovir or valacyclovir for one year post transplant significantly reduces reactivation compared to no therapy (9\% vs. $25 \%, p<0.001$ ) [48]. Patients receiving T-cell-depleting agents (proteasome inhibitors, purine analogs, and prednisone $\geq 1 \mathrm{mg} / \mathrm{kg} /$ day) are also at risk for VZV infection. Antiviral prophylaxis is continued until the immunosuppressive therapy is completed [49]. Recommendations for CLL patients receiving alemtuzumab include continuing VZV prophylaxis for 2 months beyond completion of treatment or when the CD4+ cell count is above 200 cells $/ \mu \mathrm{L}$.

\subsubsection{Cytomegalovirus (CMV)}

Reactivation and infection with CMV occur in allogeneic hematopoietic transplant recipients and patients receiving alemtuzumab treatment [50]. Screening test includes weekly quantitative CMV testing (CMV DNA by polymerase chain reaction (PCR) or CMV pp65 antigen from peripheral blood leukocytes). CMV blood testing is done for at least six months after allogeneic HSCT. Patient receiving alemtuzumab therapy should undergo surveillance during treatment and for at least 2 months after completion of treatment. Preemptive therapy for patients with CMV viremia is recommended rather than administering toxic antiviral prophylaxis. Treatment is continued for 2 weeks or until CMV viremia is no longer detectable. First-line therapy includes intravenous ganciclovir or oral valganciclovir. Ganciclovir is associated with bone marrow suppression. Second-line option foscarnet is nephrotoxic. Both acyclovir and valacyclovir are less toxic and also less active than ganciclovir. 


\section{Management of febrile neutropenia}

\subsection{Definitions}

Fever is defined as a single oral temperature of $\geq 38.3^{\circ} \mathrm{C}\left(101^{\circ} \mathrm{F}\right)$ or a temperature of $\geq 38.0^{\circ} \mathrm{C}$ $\left(100.4^{\circ} \mathrm{F}\right)$ for $1 \mathrm{~h}$ or longer [24]. Neutropenia is defined as an absolute neutrophil count (ANC) of $<1,000$ cells $/ \mu \mathrm{L}$. Severe neutropenia is defined as an ANC of $<500$ cells $/ \mu \mathrm{L}$. Profound neutropenia is defined as an ANC of $<100$ cells/ $\mu$ L. Prolonged profound neutropenia (lasting $>7$ days) is likely to occur in patients undergoing induction chemotherapy for acute leukemia or after allogeneic HSCT. Functional neutropenia occurs in patients with hematologic malignancy whose circulating neutrophils have impaired phagocytosis. These patients are at risk of infection despite "normal" neutrophil counts [51].

\subsection{Initial assessment and investigations}

Febrile neutropenia is a medical emergency requiring immediate evaluation and administration of empiric broad-spectrum antibiotics within an hour of presentation [24]. The initial assessment focuses on not only determining the probable site of infection but also the patient's risk of developing serious complications and the need for vigorous resuscitation. Relevant historical information should include the chemotherapy regimen, number of chemotherapy cycles, days since receiving anticancer treatment, concomitant use of biologic agents and steroids, growth factor support, prophylactic antimicrobials, recent surgery or radiation therapy, prior infections, HIV status, and other comorbid illnesses. Laboratory evaluation should include complete blood count with differential, renal, and hepatic function tests and cultures from all potential sites including sputum, urine, stool, skin, and mucosal ulcers as clinically relevant. At least two sets of blood cultures from peripheral veins or one set each from a peripheral vein and a central venous catheter should be drawn. Chest radiographs should be evaluated in patients with signs or symptoms of lower respiratory tract infection.

\subsection{Risk stratification}

Patients with febrile neutropenia are risk stratified into high- or low-risk groups based on the probability of development of serious infection-related complications.

\subsubsection{The Talcott model}

Patients are assigned to one of four Talcott model risk groups in the first $24 \mathrm{~h}$ of presentation. In the prospective validation study of this model $(N=444)$, medical complications developed in $5 \%$ group IV patients compared to $34 \%$ in combined groups I-III $(p<.000001)$. There were no deaths in group IV patients compared to $10 \%$ deaths in combined groups I-III. Medical complications included hypotension, tachyarrhythmias, congestive heart failure, respiratory failure, serious bleeding, altered mental status, new focal neurologic changes, and intensive care admission [52] (Figure 3). 


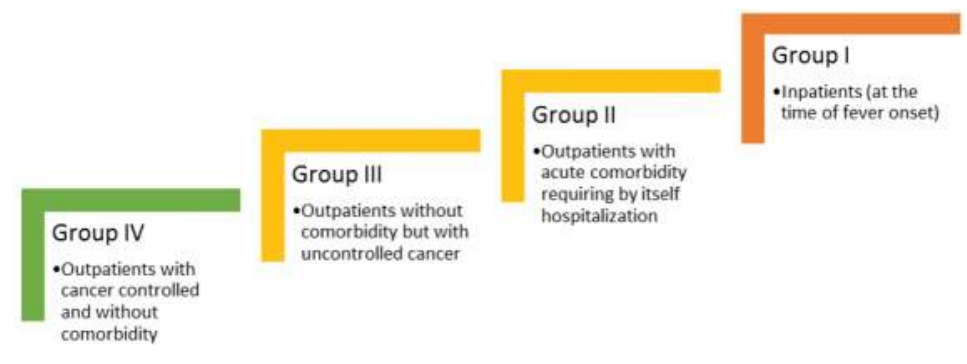

Figure 3. The Talcott model.

\subsubsection{The MASCC risk index}

The Multinational Association for Supportive Care in Cancer (MASCC) risk index uses weighted scores based on disease burden, clinical instability, age, and comorbid conditions.

The maximum theoretical MASCC score is 26. Low-risk patients have a MASCC score $\geq 21$ and mortality as low as $3 \%$. High-risk patients with MASCC score $<15$ have a mortality as high as $36 \%$. The MASCC rule does not consider the duration of anticipated neutropenia as a criteria for risk stratification [53]. Both Talcott model and MASCC index score are used to identify lowrisk febrile neutropenic patients who are suitable for outpatient management.

\subsubsection{The NCCN model [16]}

The NCCN guidelines panel considers high-risk febrile neutropenic patients as those with MASCC scores of less than 21. The panel further recommends that patients with prolonged profound neutropenia should be considered high risk, regardless of the MASCC risk index score. Other factors stratifying patients as high risk include those noted in Figure 4. These highrisk febrile neutropenic patients require hospital admission and parenteral therapy (Figure 5).

\begin{tabular}{|l|l|}
\hline Multinational Association for Supportive Care in Cancer (MASCC) & Risk-Index Score [54] \\
\hline Characteristic & W eight \\
\hline Burden of illness: & \\
$\quad$ No or mild symptoms & 5 \\
\hline Moderate symptoms & 3 \\
\hline No hypotension & 5 \\
\hline No chronic obstructive pulmonary disease & 4 \\
\hline Solid tumor or lymphoma with no previous fungal infection & 4 \\
\hline No dehydration & 3 \\
\hline Outpatient status & 3 \\
\hline Age $<60$ years & 2 \\
\hline
\end{tabular}

Figure 4. The MASCC risk index score. 


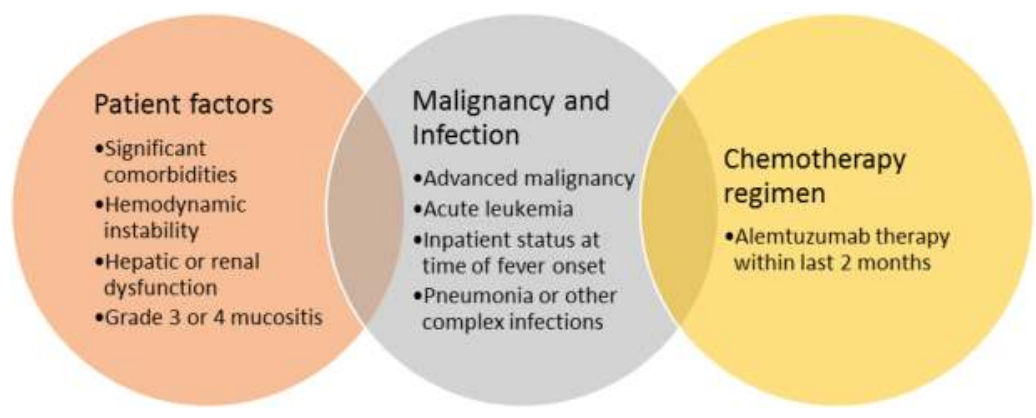

Figure 5. High-risk factors in febrile neutropenic patients.

\subsection{Outpatient therapy for low-risk patients}

Febrile neutropenic patients with MASCC scores $\geq 21$ or in Talcott group 4 are at low risk for infection-related complications provided they do not have active comorbidities or organ dysfunction. These patients can be managed safely as outpatients if they live close to a medical facility, agree to frequent clinic visits, and have 24-h caregiver support at home with easy access to telephone and transportation. Low-risk patients should receive initial doses of empirical antibacterial therapy within an hour of triage. They should then undergo a brief period of observation (at least $4 \mathrm{~h}$ ) in a medical facility to determine the suitability for outpatient management or the need for hospitalization [51].

The majority of febrile neutropenic episodes in patients receiving chemotherapy for solid tumors are low risk. Bacterial infections are the presumed culprits for unexplained fever. Fungal infections are uncommon, and reactivation of viruses is rare. In a cohort of low-risk febrile neutropenic patients $(N=757)$, unexplained febrile episodes were predominant $(58 \%)$, followed by equal frequency $(21 \%)$ of both clinically significant and microbiologically documented infections. The most common clinical sites of infection were the upper respiratory tract and skin. Among microbiologically documented infections, monomicrobial grampositive infections accounted for $49 \%$ (coagulase-negative staphylococci most frequent) followed by monomicrobial gram-negative infections (36\%, Escherichia coli predominant) and polymicrobial infections (15\%) [54].

Two randomized control trials (RCTs) of low-risk febrile neutropenic inpatients reported similar efficacy of oral ciprofloxacin plus amoxicillin/clavulanate vs. an IV regimen (ceftazidime or ceftriaxone plus amikacin) [55,56]. Ciprofloxacin monotherapy provides suboptimal coverage for gram-positive organisms including viridans group streptococci [57]. Levofloxacin is more active against gram-positive bacteria but less active than ciprofloxacin against Pseudomonas $[58,59]$. A randomized trial $(N=333)$ reported similar efficacy $(80 \%$ vs. $82 \%)$ of oral moxifloxacin compared to oral ciprofloxacin plus amoxicillin/clavulanate. Neurologic events were more common with moxifloxacin, and diarrhea was more common with the combination therapy [60]. 
Antibacterial therapy in low-risk patients with negative blood cultures is continued for at least two afebrile days after ANC recovery to $\geq 500$ cells/ $\mu \mathrm{L}$ or for five to seven days in the absence of myeloid reconstitution [61]. A meta-analysis of RCTs of low-risk febrile neutropenic patients reported that outpatient oral and parenteral antibiotics had similar efficacy (RR 0.93). The site of care (outpatient vs. inpatient) was not significantly associated with treatment failure (RR 0.81) [62]. The rate of hospital admission in patients receiving outpatient empiric therapy is in the range of $3-10 \%$.

The American Society of Clinical Oncology (ASCO) panel recommends empiric oral fluoroquinolone (ciprofloxacin or levofloxacin) plus amoxicillin/clavulanate for low-risk febrile neutropenic patients. Ciprofloxacin plus clindamycin is an alternative for penicillin-allergic patients $[24,51]$. The NCCN panel recommends moxifloxacin monotherapy for patients who may not require Pseudomonas coverage. However, patients who have received fluoroquinolone prophylaxis before fever developed are at increased risk for infection with antibiotic-resistant strains including MRSA, vancomycin-resistant Enterococcus (VRE), and ESBL-producing gramnegative bacteria. These patients therefore require hospital admission and initial management with broad-spectrum parenteral antibiotics [24] (Figure 6).

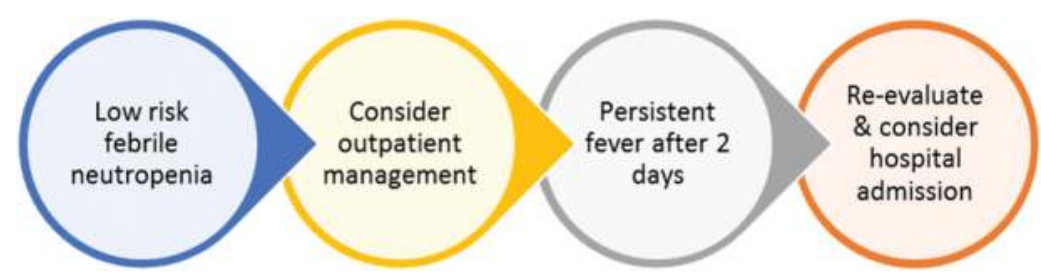

Figure 6. Management of low-risk febrile neutropenia.

\subsection{Initial empiric antibacterial therapy for high-risk patients}

Fever through periods of prolonged profound neutropenia during induction chemotherapy for acute leukemia or pre-engraftment phase of allogeneic HSCT may be due to serious or lifethreatening infections. Febrile neutropenic patients with severe comorbidities, hepatic or renal dysfunction, or MASCC scores $<21$ or in Talcott groups 1-3 are also at a high risk for infectionrelated complications. These patients should receive emergent evaluation, prompt resuscitation, and timely administration of broad-spectrum parenteral antibiotics to avoid progression to a sepsis syndrome and possibly death. A retrospective study of patients with severe sepsis reported decreased overall mortality $(19.5 \%$ vs. $33.2 \% ; p=.02)$ in patients who received antibacterial therapy within $1 \mathrm{~h}$ of presentation as opposed to latter [63].

The selection of initial antibacterial agent for high-risk febrile neutropenic patients is guided by clinical stability, recent antimicrobial use, medication allergy, potential site of infection, and susceptibility patterns of institutional pathogens. Empiric antibiotic regimens should have a broad spectrum (gram-positive and gram-negative coverage), bactericidal activity, antipseudomonal activity, and minimal toxicity. Current guidelines recommend initial monotherapy 
with antipseudomonal beta-lactam agent, such as cefepime, meropenem, imipenem-cilastatin, or piperacillin-tazobactam [16, 24]. None of these agents is superior in the empiric treatment of febrile neutropenia. However, ceftazidime monotherapy is avoided due to limited activity against viridans group streptococci and rising resistance rates among gram-negative bacteria [64].

Combination therapy is not superior to empiric monotherapy but may be associated with more adverse effects. Aminoglycoside plus antipseudomonal agent may be considered for suspected gram-negative bacteremia or sepsis syndrome or if the institution has high levels of gramnegative-resistant bacteria [65-67].

Empiric vancomycin therapy is not associated with a benefit in mortality. Nevertheless, there is concern regarding emergence of vancomycin-resistant Enterococcus (VRE) and S. aureus and increased incidence of hepatic and renal toxicity [68-71]. Current guidelines do not support its use as a routine component of the initial regimen. However, its empiric use is appropriate for suspected vascular catheter-related infection (CRI), gram-positive bacteremia, cellulitis, severe mucositis, hypotension or septic shock, pneumonia, and known colonization with MRSA or drug-resistant Streptococcus pneumoniae [72-75]. Vancomycin is usually discontinued after $48 \mathrm{~h}$ if cultures fail to grow resistant gram-positive organisms. In a randomized trial of febrile neutropenic patients with proven or suspected gram-positive infection $(N=611)$, linezolid demonstrated similar efficacy and safety when compared to vancomycin [76]. Linezolid is therefore an alternative for vancomycin-intolerant patients and vancomycinresistant gram-positive pathogens. Myelosuppression may limit its use in patients with compromised bone marrow function.

Neutropenic sepsis is a major cause of mortality. Diagnostic criteria include altered mental status, systolic hypotension of $\leq 100 \mathrm{~mm} \mathrm{Hg}$, and tachypnea of $>22$ breaths/min. These patients should receive aggressive resuscitation and monitoring in an intensive care unit. Patients in septic shock require vasopressors to maintain mean arterial pressure (MAP) $\geq 65 \mathrm{~mm} \mathrm{Hg}$ despite adequate fluid resuscitation and have a lactate level $>2 \mathrm{mmol} / \mathrm{L}$. Stress-dose steroids (50 mg hydrocortisone every six hours for 5-7 days) may benefit those with ongoing hypotension (systolic blood pressure $<90 \mathrm{~mm} \mathrm{Hg}$ for more than $1 \mathrm{~h}$ ) refractory to fluid resuscitation and vasopressor support [77]. RCTs of patients with severe sepsis demonstrated that high-dose steroids (>300 mg hydrocortisone per day) increased overall mortality and the risk of secondary infections [78-81]. The initial empiric antimicrobial regimen should include a broadspectrum beta-lactam plus aminoglycoside plus vancomycin. In addition, antifungal agents such as fluconazole or an echinocandin may be strongly considered.

The empiric broad-spectrum antibacterial should be continued until the patient is afebrile for at least 2 days and the ANC is $\geq 500$ cells/ $\mu \mathrm{L}$ on at least one occasion but is showing a consistent increasing trend. Documented infections should be managed with appropriate antimicrobials based on blood culture and susceptibility results. High-risk patients with persistent unexplained fever despite 3-5 days of antibacterial therapy should undergo assessment for undiagnosed fungal infection. Empiric coverage for Candida and/or for molds should also be considered (Figure 7). 


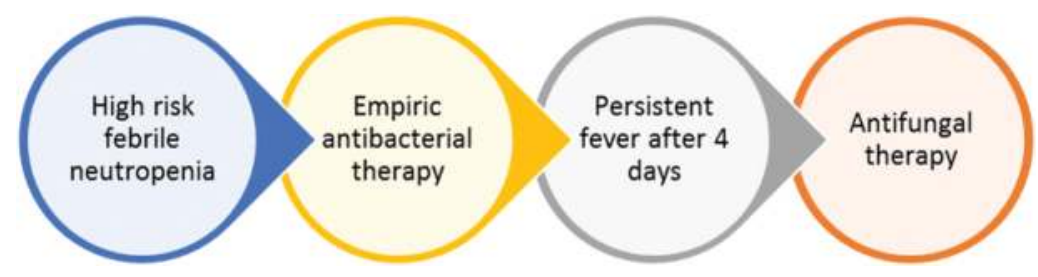

Figure 7. Management of high-risk febrile neutropenia.

\subsection{Empiric antifungal therapy}

Persistent fever refers to an episode of fever during neutropenia that does not resolve after 4 days of broad-spectrum antibacterial agents. Recurrent fever refers to a new episode of fever during neutropenia that occurs $>2$ days after resolution of a first fever while continuing broadspectrum antibacterial therapy. Patients with prolonged neutropenia with persistent or recurrent fever are at increased risk for life-threatening infections with yeasts or molds. Early detection of these invasive fungal diseases (IFDs) is challenging because of limited sensitivity and specificity of clinical presentation and investigative modalities. As an example, the mere isolation of Candida spp. from sputum, urine, or stool samples ascertains colonization only and is not indicative of invasive infection requiring treatment [82].

Definitive diagnosis of IFD requires histological evidence of deep tissue invasion or positive culture from normally sterile sites. Whereas histopathology has the ability to make organismspecific diagnoses in only a few cases, the results of fungal cultures may not be available in a timely fashion for clinical decisions. Furthermore, repeated biopsies and microbiologic samplings may be difficult to obtain in critically ill neutropenic patients. Although molecular diagnostics have the potential for increased sensitivity and a rapid turnaround time, it lacks the ability to differentiate invasive infection from colonization or contamination. The detection of fungal-specific antibodies also does not consistently differentiate between previous exposure and active disease.

Fungal antigen detection assays target components of the fungal cell wall that are shed during fungal growth. The $\beta$-(1-3)-D glucan (BDG) test detects Candida, Aspergillus, Pneumocystis, and Fusarium species in serum specimens. It has a sensitivity of $63-90 \%$ and specificity greater than $95 \%$. False-positive results may occur in patients on hemodialysis and those receiving intravenous immunoglobulin. The galactomannan (GM) assay detects Aspergillus species in both serum and bronchoalveolar lavage (BAL) specimens. It has a sensitivity of $70-89 \%$ and specificity of $85-92 \%$ in patients with hematologic malignancies [83]. BAL testing is more sensitive than serum testing in patients with invasive pulmonary aspergillosis [84]. Falsepositive results have been noted with other filamentous fungi. While the BDG assay is capable of detecting a broad range of fungi, both serum BDG and GM assays have similar sensitivities for Aspergillus species [85].

"Empiric" antifungal coverage is administered to patients without an identified fever source. 
Two RCTs of patients with persistent febrile neutropenia showed that the addition of empiric amphotericin B (on day 4 of fever or on day 7) to continued antibacterial regimen reduced the frequency of IFD [86, 87]. Initiation of antifungal agents after 4-7 days of persistent fever thus became the standard of care. Amphotericin B (AMB) use is limited by infusion reactions and renal toxicity. Subsequent studies have therefore focused on identifying safer and equally effective alternatives. Lipid formulations of amphotericin B (L-AMB) are as effective but less nephrotoxic [88]. Fluconazole lacks activity against molds (Aspergillus spp.) [89, 90]. Itraconazole has similar efficacy as AMB but less toxicity [91]. It should be used with caution in patients with reduced ejection fraction or heart failure. Intravenous formulation is not available in the United States. Erratic oral bioavailability precludes its use as an empiric agent. Voriconazole is superior to L-AMB (fewer breakthrough fungal infections). Isavuconazole is non-inferior to voriconazole with improved tolerability and safety. Caspofungin has similar efficacy as LAMB (Figure 8).

\begin{tabular}{|c|c|c|c|c|c|c|c|}
\hline \multirow[b]{2}{*}{ Author } & \multirow[b]{2}{*}{$\mathbf{N}$} & \multicolumn{2}{|c|}{ Study drugs } & \multicolumn{2}{|c|}{ Response Rate \% } & \multicolumn{2}{|c|}{$\underline{151 \%}$} \\
\hline & & Arm 1 & $\operatorname{Arm} 2$ & Arm 1 & Arm 2 & Arm 1 & $\operatorname{Arm} 2$ \\
\hline Walsh TJ[102] & 687 & AMB & L-AMB & 49 & 50 & 8.7 & 5.0 \\
\hline Boogaerts M [103] & 384 & AMB & Itraconazole & 38 & 47 & 2.7 & 2.7 \\
\hline Walsh TJ [104] & 937 & L-AMB & Voriconazole & 31 & 26 & 5.0 & $\begin{array}{c}1.9 \\
p<0.05\end{array}$ \\
\hline Walsh TJ[105] & 1095 & L-AMB & Caspofungin & 34 & 34 & 4.3 & 5.2 \\
\hline
\end{tabular}

Figure 8. Alternatives to empirical amphotericin B (AMB); L-AMB, lipid formulations of amphotericin B.

"Preemptive" antifungal treatment is administered only when the evidence of IFD is suggested by positive fungal biomarker and/or high-resolution imaging (CT chest/sinus) results. In a meta-analysis of nine published studies of high-risk patients presenting with persistent febrile neutropenia, diagnostic-driven strategy significantly reduced antifungal use (RR 0.48, $95 \%$ CI $0.27-0.85$ ) and cost without increasing IFD-related mortality (RR 0.82, $95 \%$ CI 0.36-1.87) or overall mortality (RR 0.95, $95 \%$ CI 0.46-1.99) [92]. Candida and Aspergillus are the most common fungal pathogens causing invasive disease. The widespread use of azole prophylaxis has substantially decreased the incidence of invasive candidiasis (IC) in comparison to Aspergillus and other molds. Serum BDG test is a useful screening tool for both Candida and Aspergillus species. The Fungitell (BDG) assay has a positive cutoff value of $>80 \mathrm{pg} / \mathrm{mL}$. Though a negative test result does not rule out the diagnosis of IFD, a false-positive result may occur in patients with mucositis whose gastrointestinal tract is colonized with Candida. Serum GM (cutoff optical density index $[\mathrm{ODI}]>0.5$ ) is the current gold standard for detection of invasive aspergillosis (IA). The sensitivity of this assay is significantly reduced in patients receiving anti-mold prophylaxis. Combining BG and GM assays improves the diagnosis of IA [93].

Aspergillus DNAemia may precede the release of fungal GM into the bloodstream. However, Aspergillus polymerase chain reaction (PCR) testing has not been widely implemented due to a lack of standardization. A randomized trial reported that combined serum GM and Asper- 
gillus PCR monitoring leads to an earlier diagnosis of invasive aspergillosis [94]. A metaanalysis of thirteen studies $(N=1670)$ showed that the absence of serum GM and Aspergillus PCR-positive test may obviate the need for antifungal agents with a negative predictive value of $100 \%$ [95].

The echinocandins (caspofungin, micafungin, and anidulafungin) are fungicidal against most Candida spp. with similar efficacy [96-99]. Voriconazole is the treatment of choice for invasive aspergillosis. Isavuconazole is non-inferior to voriconazole with fewer side effects [100].

The European Organization for Research and Treatment of Cancer (EORTC) is completing accrual $(N=556)$ of a phase 3 prospective trial (NCT01288378) comparing empiric and preemptive caspofungin therapy in patients with AML or MDS. The results of this study should clarify the utility of diagnostic testing and the efficacy of preemptive strategies in patients receiving antifungal prophylaxis with fluconazole (Figures 9 and 10).

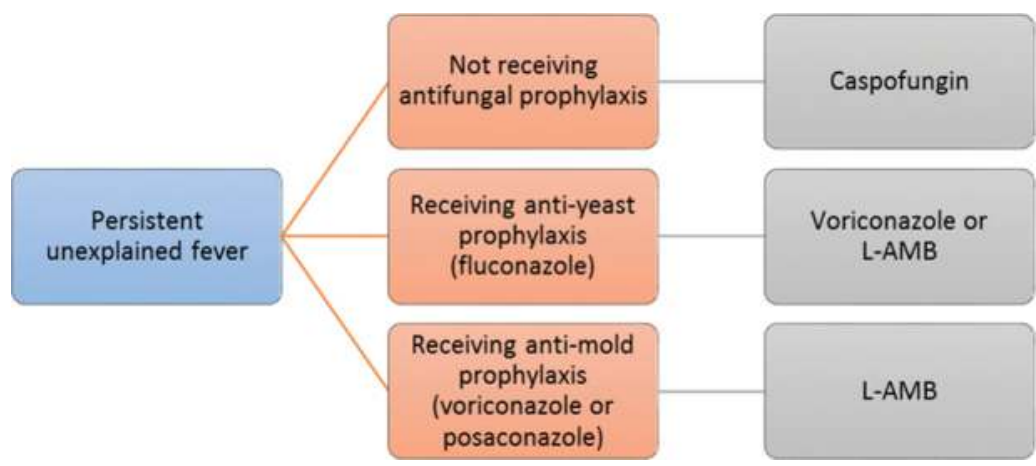

Figure 9. Management of persistent febrile neutropenia. L-AMB, lipid formulations of amphotericin B.

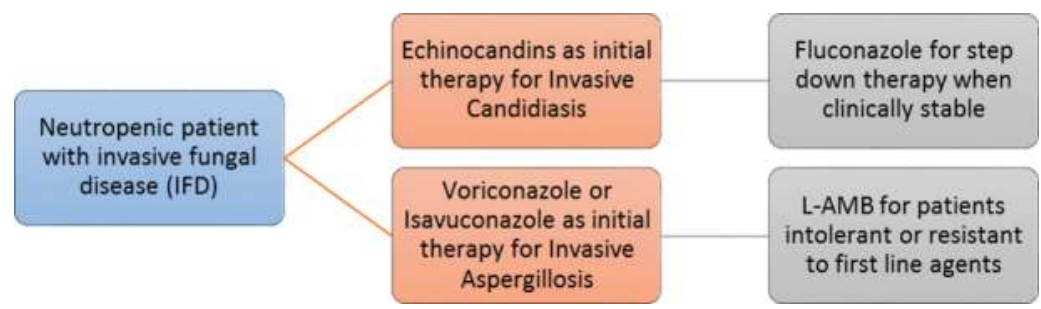

Figure 10. Management of febrile neutropenia with invasive fungal disease. L-AMB, lipid formulations of amphoteri$\operatorname{cin} \mathrm{B}$.

\subsection{Vascular catheter-related infections}

Central venous catheters (Hickman or Mediport) are frequently used in patients undergoing cancer treatment. Neutropenic patients are at increased risk for vascular catheter-related 
infections (CRI). The hub/lumen of the catheter is the major site of colonization and source of infection. The differential time to positivity (DTP) of $120 \mathrm{~min}$ or more between centrally and peripherally drawn blood cultures is indicative of catheter-related bacteremia. Common pathogens include coagulase-negative staphylococci, S. aureus, and Candida spp.

Febrile neutropenic patients with clinical signs of CRI should receive empiric antipseudomonal beta-lactam agent plus vancomycin. Catheter removal should be strongly considered for tunnel or port-pocket infections; septic phlebitis; septic shock; endocarditis; bacteremia due to $S$. aureus, $P$. aeruginosa, or Candida spp.; and persistent bloodstream infection despite $\geq 72 \mathrm{~h}$ of therapy. Catheter removal is not required for coagulase-negative staphylococci bacteremia. Antimicrobial therapy is modified after availability of blood culture and susceptibility results. Antibacterial agents should be administered for a minimum of 14 days following catheter removal and clearance of blood cultures. Prolonged treatment for 4-6 weeks is recommended for bacteremia complicated with deep tissue infection, endocarditis, septic thrombosis, or persistent bacteremia or fungemia occurring $>72 \mathrm{~h}$ after catheter removal [24].

\section{Refining infection control during cancer care}

Preventing infection in cancer patients is a comprehensive initiative led by Centers for Disease Control and Prevention to reduce infections in patients with cancer. PreventCancerInfections.org is a website that provides information about neutropenia, signs and symptoms of infections, and methods to control them. Basic infection control and prevention plan is a tool for outpatient oncology facilities that outlines infection control policies and procedures. Standard precautions refer to the minimum measures to prevent infection including hand and respiratory hygiene, proper use of gowns and gloves, injection safety, medication storage and handling, and cleaning and disinfection of devices and environmental surfaces. Transmissionbased precautions supplement standard precautions when managing potentially infectious patients. Medical providers should perform hand hygiene before and after contact with the patients. The examination room should be cleaned and disinfected before using it for another patient.

Home infection prevention measures include avoiding contact with sick people or sharing personal items, keeping household surfaces clean, consuming clean and properly cooked food, and practicing good oral, dental, and skin hygiene. Patients should be instructed to contact their provider immediately in the event of fever, redness, swelling, or drainage from surgical and vascular catheter sites. Annual influenza vaccination with the inactivated virus is recommended for all caregivers.

High-risk patients with febrile neutropenia require hospital admission. Allogeneic HSCT recipients should be confined in private rooms with $>12$ air exchanges/h and high-efficiency particulate air filtration. Non-transplant patients do not require a private room. Household pets, fresh flowers, and plants should not be allowed in the rooms [24]. Patients should take daily showers, maintain good health hygiene, and eat well-cooked foods. Rectal thermometers, enemas, suppositories, and rectal examinations should be avoided during periods of neutro- 
penia. Healthcare workers and visitors with symptomatic infections should avoid contact with the neutropenic patient. Hospitals should conduct periodic risk assessment of multidrugresistant organism acquisition and transmission.

\section{Conclusions}

Infection is an important cause of morbidity and mortality in cancer patients. Febrile neutropenia is a frequent and expensive complication of myelosuppressive chemotherapy. Evidencebased guidelines provide strong recommendations for the empiric management of initial fever and persistent fever. The management of febrile patients receiving anti-yeast or anti-mold prophylaxis is still evolving. The best management of recurrent fever remains unanswered. Judicious use of antimicrobial prophylaxis is an important infection prevention strategy. Hand hygiene, contact precautions, and disinfecting patient-care equipment remain crucial approaches for preventing the spread of infections in medical facilities. The repertoire of new medications for the treatment of cancer is continually expanding. Physicians should be vigilant of the immunosuppressive potential and the risk of opportunistic infections associated with the use of these newer biologics.

\section{Author details}

Deepjot Singh ${ }^{1,3^{*}}$ and Robert A. Bonomo ${ }^{2,3}$

*Address all correspondence to: deepjot.singh@case.edu

1 Hematology Oncology, Medical Service, Louis Stokes Cleveland Department of Veterans Affairs Medical Center, Cleveland, USA

2 Infectious Diseases Sections, Medical Service, Louis Stokes Cleveland Department of Veterans Affairs Medical Center, Cleveland, USA

3 Department of Medicine, Case Western Reserve University, Cleveland, USA

\section{References}

[1] Preventing Infections in Cancer Patients. Centers for Disease Control. Nov. 19, 2015. Retrieved from http://www.cdc.gov/cancer/dcpc/resources/features/PreventInfections/index.htm

[2] Dinan MA, Hirsch BR, Lyman GH. Management of chemotherapy-induced neutropenia: measuring quality, cost, and value. J Natl Compr Canc Netw. 2015;13(1):e1-e7. 
[3] Saral R, Burns WH, Prentice HG. Herpes virus infections: clinical manifestations and therapeutic strategies in immunocompromised patients. Clin Haematol. 1984;13:645660.

[4] Funakoshi T, Suzuki M, Muss HB. Infection risk in breast cancer patients treated with trastuzumab: a systematic review and meta-analysis. Breast Cancer Res Treat. 2015;149(2):321-330.

[5] Garcia CA, Wu S. Risk of serious infection with mTOR inhibitors everolimus and temsirolimus in the treatment of cancer: a meta-analysis of randomized controlled trials. J Clin Oncol. 2014;32s:35s.

[6] Ma Q, Gu LY, Ren YY, et al. Increased risk of severe infections in cancer patients treated with vascular endothelial growth factor receptor tyrosine kinase inhibitors: a metaanalysis. Onco Targets Ther. 2015;8:2361-2374.

[7] Sandherr, M, Hentrich, M, von Lilienfeld-Toal, M. Antiviral prophylaxis in patients with solid tumours and haematological malignancies-update of the Guidelines of the Infectious Diseases Working Party (AGIHO) of the German Society for Hematology and Medical Oncology (DGHO). Ann Hematol. 2015;94(9): 1441-1450.

[8] Mitka M. FDA: increased HBV reactivation risk with ofatumumab or rituximab. JAMA. 2013;310(16):1664.

[9] Huang H, Li X, Zhu J, et al. Entecavir vs lamivudine for prevention of hepatitis $B$ virus reactivation among patients with untreated diffuse large B-cell lymphoma receiving R-CHOP chemotherapy: a randomized clinical trial. JAMA. 2014;312:25212530.

[10] Bradley AM, Devine M, DeRemer D. Brentuximab vedotin: an anti-CD30 antibodydrug conjugate. Am J Health Syst Pharm. 2013;70(7):589-597.

[11] Darzalex (Daratumumab). Package insert, https://www.janssenmd.com/pdf/darzalex/ DARZALEX_PI.pdf

[12] Montillo M, Ricci F, Miqueleiz S, et al. Alemtuzumab in the treatment of fludarabine refractory B-cell chronic lymphocytic leukemia (CLL). Biologics. 2008;2(1):41-52.

[13] Markham A. Elotuzumab: first global approval. Drugs. 2016;76(3):397-403.

[14] Harrison C, Mesa R, Ross D, et al. Practical management of patients with myelofibrosis receiving ruxolitinib. Expert Rev Hematol. 2013;6(5):511-523.

[15] Santos FP, Verstovsek S. Efficacy of ruxolitinib for myelofibrosis. Expert Opin Pharmacother. 2014;15(10):1465-1473.

[16] National Comprehensive Cancer Network (NCCN). Clinical Practice Guidelines in Oncology. Prevention and treatment of cancer-related infections. Version 2. 2015. http:// www.nccn.org (Accessed on February 12, 2016). 
[17] Engels EA, Lau J, Barza M. Efficacy of quinolone prophylaxis in neutropenic cancer patients: a meta-analysis. J Clin Oncol. 1998;16:1179-1187.

[18] Gafter-Gvili A, Fraser A, Paul M, et al. Antibiotic prophylaxis for bacterial infections in afebrile neutropenic patients following chemotherapy. Cochrane Database Syst Rev. 2012;1:CD004386.

[19] Bucanev G, Micozzi A, Menichetti F, et al. Levofloxacin to prevent bacterial infection in patients with cancer and neutropenia. N Engl J Med. 2005;353:977-987.

[20] Cullen M, Steven N, Billingham L, et al. Antibacterial prophylaxis after chemotherapy for solid tumors and lymphomas. N Engl J Med. 2005;353 (10):988.

[21] Razonable R, Litzow MR, Khaliq Y, et al. Bacteremia due to viridans group Streptococci with diminished susceptibility to Levofloxacin among neutropenic patients receiving levofloxacin prophylaxis. Clin Infect Dis. 2002;34(11):1469-1474.

[22] Cook PP, Catrou P, Gooch M, et al. Effect of reduction in ciprofloxacin use on prevalence of methicillin-resistant Staphylococcus aureus rates within individual units of a tertiary care hospital. J Hosp Infect. 2006;64(4):348-351.

[23] Loo VG, Poirier L, Miller MA, et al. A predominantly clonal multi-institutional outbreak of Clostridium difficile-associated diarrhea with high morbidity and mortality. N Engl J Med. 2005;353:2442-2449.

[24] Freifeld AG, Bow EJ, Sepkowitz KA, et al. Clinical practice guideline for the use of antimicrobial agents in neutropenic patients with cancer: 2010 Update by the Infectious Diseases Society of America. Infectious Diseases Society of America, Clin Infect Dis. 2011;52(4):e56.

[25] Rotstein C, Bow EJ, Laverdiere M, et al. Randomized placebo-controlled trial of fluconazole prophylaxis for neutropenic cancer patients: benefit based on purpose and intensity of cytotoxic therapy. The Canadian Fluconazole Prophylaxis Study Group. Clin Infect Dis. 1999;28:331-340.

[26] Koh LP, Kurup A, Goh YT, et al. Randomized trial of fluconazole versus low-dose amphotericin B in prophylaxis against fungal infections in patients undergoing hematopoietic stem cell transplantation. Am J Hematol. 2002;71:260-267.

[27] Ethier MC, Science M, Beyene J, et al. Mould-active compared with fluconazole prophylaxis to prevent invasive fungal diseases in cancer patients receiving chemotherapy or haematopoietic stem-cell transplantation: a systematic review and metaanalysis of randomised controlled trials. Br J Cancer. 2012;106(10):1626-1637.

[28] Vardakas KZ, Michalopoulos A, Falagas ME. Fluconazole versus itraconazole for antifungal prophylaxis in neutropenic patients with haematological malignancies: a meta-analysis of randomised-controlled trials. $\mathrm{Br} J$ Haematol. 2005;131(1): $22-28$. 
[29] Wang J, Zhan P, Zhou R, et al. Prophylaxis with itraconazole is more effective than prophylaxis with fluconazole in neutropenic patients with hematological malignancies. Med Oncol. 2010;27(4):1082-1088.

[30] Marks DI, Pagliuca A, Kibbler CC, et al. IMPROVIT Study Group. Voriconazole versus itraconazole for antifungal prophylaxis following allogeneic haematopoietic stem-cell transplantation. Br J Haematol. 2011;155(3):318-327.

[31] Cornely OA, Maertens J, Winston DJ, et al. Posaconazole vs. fluconazole or itraconazole prophylaxis in patients with neutropenia. N Engl J Med. 2007;356(4):348-359.

[32] Mattiuzzi GN, Alvarado G, Giles FJ, et al. Open-label, randomized comparison of itraconazole versus caspofungin for prophylaxis in patients with hematologic malignancies. Antimicrob Agents Chemother. 2006;50(1):143-147.

[33] Wingard JR, Carter SL, Walsh TJ, et al. Blood and Marrow Transplant Clinical Trials Network. Randomized, double-blind trial of fluconazole versus voriconazole for prevention of invasive fungal infection after allogeneic hematopoietic cell transplantation. Blood. 2010;116(24):5111-5118.

[34] Huang X, Chen H, Han M, et al. Multicenter, randomized, open-label study comparing the efficacy and safety of micafungin versus itraconazole for prophylaxis of invasive fungal infections in patients undergoing hematopoietic stem cell transplant. Biol Blood Marrow Transplant. 2012;18(10):1509-1516.

[35] Ullmann AJ, Lipton JH, Vesole DH, et al. Posaconazole or fluconazole for prophylaxis in severe graft-versus-host disease. N Engl J Med. 2007;356(4):335-347.

[36] Chaftari AM, Hachem RY, Ramos E, et al. Comparison of posaconazole versus weekly amphotericin B lipid complex for the prevention of invasive fungal infections in hematopoietic stem-cell transplantation. Transplantation. 2012;94(3):302-308.

[37] Stern A, Green H, Paul M, et al. Prophylaxis for Pneumocystis pneumonia (PCP) in non-HIV immunocompromised patients. Cochrane Database Syst Rev. 2014;10:CD005590.

[38] Marras TK, Sanders K, Lipton JH, et al. Aerosolized pentamidine prophylaxis for Pneumocystis carinii pneumonia after allogeneic marrow transplantation. Transpl Infect Dis. 2002;4(2):66-74.

[39] Sangiolo D, Storer B, Nash R, et al. Toxicity and efficacy of daily dapsone as Pneumocystis jiroveci prophylaxis after hematopoietic stem cell transplantation: a case-control study. Biol Blood Marrow Transplant. 2005;11(7):521-529.

[40] Eliakim-Raz N, Vinograd I, Zalmanovici TA, et al. Influenza vaccines in immunosuppressed adults with cancer. Cochrane Database Syst Rev. 2013;10:CD008983.

[41] Lorry R, Myron L, Per L, et al. 2013 IDSA clinical practice guideline for vaccination of the immunocompromised host. Clin Infect Dis. 2014;58(3):e44-e100. 
[42] Engelhard D, Mohty B, de la Camara R, et al. European guidelines for prevention and management of influenza in hematopoietic stem cell transplantation and leukemia patients: summary of ECIL-4 (2011), on behalf of ECIL, a joint venture of EBMT, EORTC, ICHS, and ELN. Transpl Infect Dis. 2013;15:219-232.

[43] Reddy KR, Beavers KL, Hammond SP, et al. American Gastroenterological Association I. American Gastroenterological Association Institute guideline on the prevention and treatment of hepatitis B virus reactivation during immunosuppressive drug therapy. Gastroenterology. 2015;148:215-219, quiz e16-e17.

[44] Marinone C, Mestriner M. HBV disease: HBsAg carrier and occult B infection reactivation in haematological setting. Dig Liver Dis. 2011;43(Suppl 1):S49-S56.

[45] Zaia J, Baden L, Boeckh MJ, et al. Viral disease prevention after hematopoietic cell transplantation. Bone Marrow Transplant. 2009;44(8):471-482.

[46] Yahav D, Gafter-Gvili A, Muchtar E, et al. Antiviral prophylaxis in haematological patients: systematic review and meta-analysis. Eur J Cancer. 2009;45(18):3131-3148.

[47] Keating M, Coutré S, Rai K, et al. Management guidelines for use of alemtuzumab in B-cell chronic lymphocytic leukemia. Clin Lymphoma. 2004;4(4):220-227.

[48] Erard V, Guthrie KA, Varley C et al. One-year acyclovir prophylaxis for preventing varicella-zoster virus disease after hematopoietic cell transplantation: no evidence of rebound varicella-zoster virus disease after drug discontinuation. Blood. 2007;110(8): 3071-3077.

[49] Tomblyn M, Chiller T, Einsele H, et al. Guidelines for preventing infectious complications among hematopoietic cell transplantation recipients: a global perspective. Biol Blood Marrow Transplant. 2009;15(10):1143.

[50] George B, Pati N, Gilroy N, et al. Pre-transplant cytomegalovirus (CMV) serostatus remains the most important determinant of CMV reactivation after allogeneic hematopoietic stem cell transplantation in the era of surveillance and preemptive therapy. Transpl Infect Dis. 2010;12(4):322-329.

[51] Flowers CR, Seidenfeld J, Bow EJ et al. Antimicrobial prophylaxis and outpatient management of fever and neutropenia in adults treated for malignancy: American Society of Clinical Oncology Clinical Practice Guideline. JCO. 2013;31(3):794-810.

[52] Talcott JA, Siegel RD, Finberg R et al. Risk assessment in cancer patients with fever and neutropenia: a prospective, two-center validation of a prediction rule. J Clin Oncol. 1992;10:316-322.

[53] Klastersky J, Paesmans M, Rubenstein EB et al. The Multinational Association for Supportive Care in Cancer risk index: a multinational scoring system for identifying low-risk febrile neutropenic cancer patients. J Clin Oncol. 2000;18(16): 3038-3051. 
[54] Kamana M, Escalante C, Mullen CA, et al. Bacterial infections in low-risk, febrile neutropenic patients. Cancer. 2005;104(2):422-426.

[55] Freifeld A, Marchigiani D, Walsh T, et al. A double-blind comparison of empirical oral and intravenous antibiotic therapy for low-risk febrile patients with neutropenia during cancer chemotherapy. N Engl J Med. 1999;341(5):305-311.

[56] Kern WV, Cometta A, De Bock R, et al. Oral versus intravenous empirical antimicrobial therapy for fever in patients with granulocytopenia who are receiving cancer chemotherapy. International Antimicrobial Therapy Cooperative Group of the European Organization for Research and Treatment of Cancer. N Engl J Med. 1999;341(5):312318.

[57] Kerr KG, Armitage HT, McWhinney PH. Activity of quinolones against viridans group streptococci isolated from blood cultures of patients with haematological malignancy. Support Care Cancer. 1999;7(1):28-30.

[58] Gasink LB, Fishman NO, Weiner MG, et al. Fluoroquinolone-resistant Pseudomonas aeruginosa: assessment of risk factors and clinical impact. Am J Med. 2006;119:526.e19526.e25.

[59] Kaye KS, Kanafani ZA, Dodds AE, et al. Differential effects of levofloxacin and ciprofloxacin on the risk for isolation of quinolone-resistant Pseudomonas aeruginosa. Antimicrob Agents Chemother. 2006;50:2192-2196.

[60] Kern WV, Marchetti O, Drgona L, et al. Oral antibiotics for fever in low-risk neutropenic patients with cancer: a double-blind, randomized, multicenter trial comparing single daily moxifloxacin with twice daily ciprofloxacin plus amoxicillin/clavulanic acid combination therapy-EORTC infectious diseases group trial XV. J Clin Oncol. 2013;31(9):1149-1156.

[61] de Naurois J, Novitzky-Basso I, Gill MJ, et al. Management of febrile neutropenia: ESMO Clinical Practice Guidelines. Ann Oncol. 2010;21 (Suppl 5):v252-v256.

[62] Teuffel O, Ethier MC, Alibhai SM, et al. Outpatient management of cancer patients with febrile neutropenia: a systematic review and meta-analysis. Ann Oncol. 2011;22(11): 2358-2365.

[63] Gaieski DF, Mikkelsen ME, Band RA, et al. Impact of time to antibiotics on survival in patients with severe sepsis or septic shock in whom early goal-directed therapy was initiated in the emergency department. Crit Care Med. 2010;38:1045-1053.

[64] Tunkel AR, Sepkowitz KA. Infections caused by viridans streptococci in patients with neutropenia. Clin Infect Dis. 2002;34(11):1524-1529.

[65] Cometta A, Zinner S, de Bock R, et al. Piperacillin-tazobactam plus amikacin versus ceftazidime plus amikacin as empiric therapy for fever in granulocytopenic patients with cancer. The International Antimicrobial Therapy Cooperative Group of the 
European Organization for Research and Treatment of Cancer. Antimicrob Agents Chemother. 1995;39(2):445-452.

[66] Cordonnier C, Herbrecht R, Pico JL, et al. Cefepime/amikacin versus ceftazidime/amikacin as empirical therapy for febrile episodes in neutropenic patients: a comparative study. The French Cefepime Study Group. Clin Infect Dis. 1997;24(1):41-51

[67] Flaherty JP, Waitley D, Edlin B et al. Multicenter, randomized trial of ciprofloxacin plus azlocillin versus ceftazidime plus amikacin for empiric treatment of febrile neutropenic patients. Am J Med. 1989;87(5A):278S-282S.

[68] Edmond MB, Ober JF, Weinbaum DL, et al. Vancomycin-resistant Enterococcus faecium bacteremia: risk factors for infection. Clin Infect Dis. 1995;20(5):1126-1133.

[69] Montecalvo MA, Shay DK, Patel P, et al. Bloodstream infections with vancomycinresistant enterococci. Arch Intern Med. 1996;156(13):1458-1462.

[70] Vancomycin added to empirical combination antibiotic therapy for fever in granulocytopenic cancer patients. European Organization for Research and Treatment of Cancer (EORTC) International Antimicrobial Therapy Cooperative Group and the National Cancer Institute of Canada-Clinical Trials Group. J Infect Dis. 1991;163(5):951-958.

[71] Lin MY, Hayden MK. Methicillin-resistant Staphylococcus aureus and vancomycinresistant enterococcus: recognition and prevention in intensive care units. Crit Care Med. 2010;38(8 Suppl):S335-S344.

[72] Mermel LA, Allon M, Bouza E, et al. Clinical practice guidelines for the diagnosis and management of intravascular catheter-related infection: 2009 Update by the Infectious Diseases Society of America. Clin Infect Dis. 2009;49(1):1-45

[73] Dellinger RP, Levy MM, Carlet JM, et al. Surviving Sepsis Campaign: international guidelines for management of severe sepsis and septic shock: 2008. Intensive Care Med. 2008;34(1):17-60.

[74] Kaul DR, Collins CD, Hyzy RC. New developments in antimicrobial use in sepsis. Curr Pharm Des. 2008;14(19):1912-1920.

[75] Daum RS. Clinical practice. Skin and soft-tissue infections caused by methicillinresistant Staphylococcus aureus. N Engl J Med. 2007;357(4):380-390.

[76] Jaksic B, Martinelli G, Perez-Oteyza J, et al. Efficacy and safety of linezolid compared with vancomycin in a randomized, double-blind study of febrile neutropenic patients with cancer. Clin Infect Dis. 2006;42(5):597-607.

[77] Patel GP, Balk RA. Systemic steroids in severe sepsis and septic shock. Am J Respir Crit Care Med. 2012;185:133-139. 
[78] Veterans Administration Systemic Sepsis Cooperative Study Group. Effect of high-dose glucocorticoid therapy on mortality in patients with clinical signs of systemic sepsis. N Engl J Med. 1987;317(11):659-665.

[79] Bone RC, Fisher CJ Jr, Clemmer TP, et al. A controlled clinical trial of high-dose methylprednisolone in the treatment of severe sepsis and septic shock. N Engl J Med. 1987;317(11):653-658.

[80] Cronin L, Cook DJ, Carlet J, et al. Corticosteroid treatment for sepsis: a critical appraisal and meta-analysis of the literature. Crit Care Med. 1995;23(8):1430-1439.

[81] Slotman GJ, Fisher CJ Jr, Bone RC, et al. Detrimental effects of high-dose methylprednisolone sodium succinate on serum concentrations of hepatic and renal function indicators in severe sepsis and septic shock. The Methylprednisolone Severe Sepsis Study Group. Crit Care Med. 1993;21(2):191-195.

[82] Cornely OA, Bassetti M, Calandra T, et al. ESCMID* guideline for the diagnosis and management of Candida diseases 2012: non-neutropenic adult patients. Clin Microbiol Infect. 2012;18(Suppl 7):19-37.

[83] Pfeiffer CD, Fine JP, Safdar N. Diagnosis of invasive aspergillosis using a galactomannan assay: a meta-analysis. Clin Infect Dis. 2006;42(10):1417-1427

[84] Fisher CE, Stevens AM, Leisenring W, et al. Independent contribution of bronchoalveolar lavage and serum galactomannan in the diagnosis of invasive pulmonary aspergillosis. Transpl Infect Dis. 2014;16(3):505-510.

[85] Marty FM, Koo S. Role of (1-3)-beta-d-glucan in the diagnosis of invasive aspergillosis. Med Mycol. 2009;47(Suppl 1):S233-S240.

[86] Pizzo PA, Robichaud KJ, Gill FA, et al. Empiric antibiotic and antifungal therapy for cancer patients with prolonged fever and granulocytopenia. Am J Med. 1982;72(1):101111.

[87] Empiric antifungal therapy in febrile granulocytopenic patients. EORTC International Antimicrobial Therapy Cooperative Group. Am J Med. 1989;86(6Pt1):668-672.

[88] Walsh TJ, Finberg RW, Arndt C, et al. Liposomal amphotericin B for empirical therapy in patients with persistent fever and neutropenia. National Institute of Allergy and Infectious Diseases Mycoses Study Group. N Engl J Med. 1999;340(10):764-771.

[89] Viscoli C, Castagnola E, Van Lint MT, et al. Fluconazole versus amphotericin B as empirical antifungal therapy of unexplained fever in granulocytopenic cancer patients: a pragmatic, multicentre, prospective and randomised clinical trial. Eur J Cancer. 1996;32A(5):814-820.

[90] Winston DJ, Hathorn JW, Schuster MG, et al. A multicenter, randomized trial of fluconazole versus amphotericin B for empiric antifungal therapy of febrile neutropenic patients with cancer. Am J Med. 2000;108(4):282-289. 
[91] Boogaerts M, Winston DJ, Bow EJ, et al. Intravenous and oral itraconazole versus intravenous amphotericin B deoxycholate as empirical antifungal therapy for persistent fever in neutropenic patients with cancer who are receiving broad-spectrum antibacterial therapy. A randomized, controlled trial. Ann Intern Med. 2001;135(6):412-422.

[92] Fung M, Kim J, Marty FM, et al. Meta-analysis and cost comparison of empirical versus pre-emptive antifungal strategies in hematologic malignancy patients with high-risk febrile neutropenia. PLoS ONE. 2015;10(11):e0140930. doi: 10.1371/journal.pone. 0140930

[93] Pazos, C, Ponton J, Del Palacio A. Contribution of $(1 \rightarrow 3)-\beta-d-g l u c a n$ chromogenic assay to diagnosis and therapeutic monitoring of invasive aspergillosis in neutropenic adult patients: a comparison with serial screening for circulating galactomannan. J Clin Microbiol 2005;43:299-305.

[94] Aguado JM, Vasquez L, Fernandez-Ruiz M, et al. Serum galactomannan versus a combination of galactomannan and polymerase chain reaction-based Aspergillus DNA detection for early therapy of invasive aspergillosis in high-risk hematological patients: a randomized controlled trial. Clin Infect Dis. 2015;60:405-414. doi: 10.1093/cid/ciu833. pmid: 25336623

[95] Arvanitis M, Anagnostou T, Mylonakis E. Galactomannan and polymerase chain reaction-based screening for invasive aspergillosis among high-risk hematology patients: a diagnostic meta-analysis. Clin Infect Dis. 2015;61(8):1263-1272.

[96] Mora-Duarte J, Betts R, Rotstein C, et al. Comparison of caspofungin and amphotericin B for invasive candidiasis. N Engl J Med. 2002;347(25):2020-2029.

[97] Kuse ER, Chetchotisakd P, da Cunha CA, et al. Micafungin versus liposomal amphotericin B for candidaemia and invasive candidosis: a phase III randomized double-blind trial. Lancet. 2007;369(9572):1519-1527.

[98] Reboli AC, Shorr AF, Rotstein C, et al. Anidulafungin compared with fluconazole for treatment of candidemia and other forms of invasive candidiasis caused by Candida albicans: a multivariate analysis of factors associated with improved outcome. BMC Infect Dis. 2011;11:261-269.

[99] Pappas PG, Rotstein CM, Betts RF, et al. Micafungin versus caspofungin for treatment of candidemia and other forms of invasive candidiasis. Clin Infect Dis. 2007;45(7):883893.

[100] Maertens J, Patterson TF, Rahav G, et al. A phase 3 randomized, double-blind trial evaluating isavuconazole vs voriconazole for the primary treatment of invasive fungal disease caused by Aspergillus spp. or other filamentous fungi (SECURE). 24th ECCMID. Barcelona, Spain, 10-13 May, 2014. 
[101] Walsh TJ, Pappas P, Winston DJ, et al. Voriconazole compared with liposomal amphotericin $\mathrm{B}$ for empirical antifungal therapy in patients with neutropenia and persistent fever. N Engl J Med. 2002;346(4):225-234.

[102] Walsh TJ, Teppler H, Donowitz GR, et al. Caspofungin versus liposomal amphotericin $\mathrm{B}$ for empirical antifungal therapy in patients with persistent fever and neutropenia. $\mathrm{N}$ Engl J Med. 2004;351(14):1391-1402. 
